\title{
SYNTHESIS AND ANTIBACTERIAL EVALUATION OF NEW PYRROLO[3',4':3,4]PYRROLO[1,2-a]QUINOLINE AND PYRROLO[3',4':3,4]PYRROLO[2,1-a]ISOQUINOLINE DERIVATIVES
}

\author{
CRISTINA M. AL MATARNEH ${ }^{a}$, MONICA C. SARDARU \\ MIRCEA O. APOSTU, ${ }^{\text {, IRINA ROSCA }}{ }^{\mathrm{b}}$, CATALINA I. CIOBANU, \\ IONEL I. MANGALAGIU ${ }^{a}$, RAMONA DANAC ${ }^{\mathrm{a}, *}$
}

\begin{abstract}
Two series of new fused pyrrolo[3',4':3,4]pyrrolo[1,2-a]quinoline and pyrrolo[3',4':3,4]pyrrolo[2,1-a]isoquinoline derivatives were synthesized and evaluated for the antimicrobial activity. The synthetic approach involves cycloimmonium ylides as 1,3-dipol intermediates. The structures of all synthesized compounds were proved by analytical and spectroscopic data. Crystal structure of compound 11a has been also determined by single crystal XRD. The synthesized compounds were evaluated for their expected antimicrobial activity against Staphylococcus aureus ATCC25923, Escherichia coli ATCC25922 and Candida albicans ATCC10231, but no one showed activity against all the reference strains.
\end{abstract}

Keywords: heterocycles, cycloaddition, quinolines, isoquinolines, antibacterial

\section{INTRODUCTION}

In recent years, medicinal chemistry has been focused on the development of new potent antimicrobial agents, to overcome the problem of bacterial resistance, which became a very serious medical problem for health care systems in the entire world $[1,2]$.

In this respect, fused $\mathrm{N}$-heterocyclic systems attracted an important interest, since their molecules contain two or more widely known pharmacophoric fragments [3].

a "Al. I. Cuza" University of lasi, Faculty of Chemistry, Department of Chemistry, 11 Carol I, 700506 lasi, Romania

b Institute of Macromolecular Chemistry "Petru Poni" lasi, Romania

c "Al. I. Cuza" University of lasi, Faculty of Chemistry, Research Department, 11 Carol I, 700506 lasi, Romania

*Corresponding author: rdanac@uaic.ro 
Pyrrolo[2,1-a]isoquinolines are particular structural units in the heterocycle class, some of them occurring in biologically active compounds, the best known being lamellarine and jamtine groups of alkaloids (fig. 1). Some representatives of the group are known for their inhibitory properties of topoisomerase or HIV-1 integrase enzymes, while others showed anticancer activity, anti-inflammatory or cardiovascular properties [4].

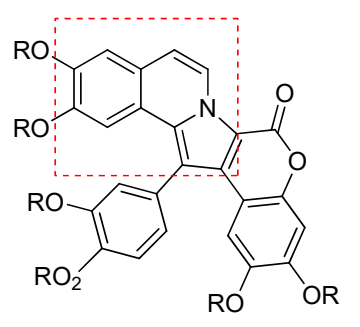

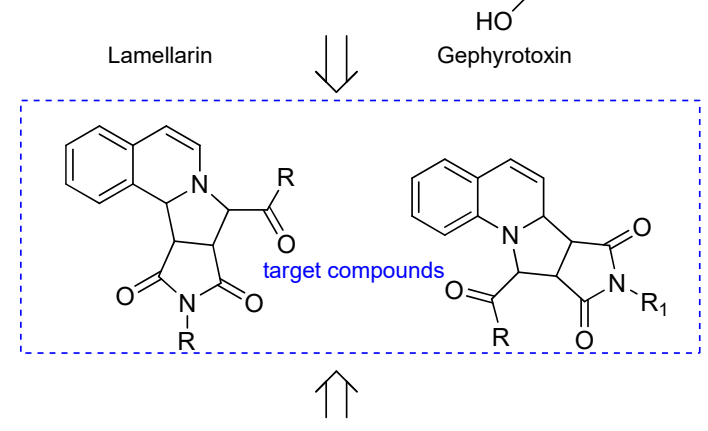<smiles>[R]C(=O)NN1C(=O)c2nc3ccccc3c([R])c2C1=O</smiles>

antibacterial activity [13]

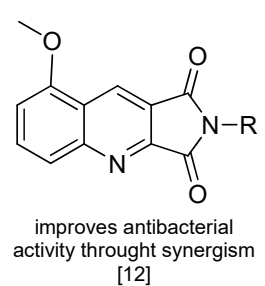

Figure 1. Design of the target compounds

Pyrrolo[1,2-a]quinolines are also present in marine alkaloids as gephyrotoxin (fig. 1) that is reported as a muscarinic antagonist and presents interesting neurological activities $[5,6]$.

For these reasons, the synthesis of pyrrolo[1,2-a]quinolines and pyrrolo[2,1-a] isoquinolines has drawn much recent research interest. Several synthetic methods for these fused $\mathrm{N}$-heterocycles have been reported, including the reactions of 2-alkylpyridine with aldehydes or a-haloketone [7], 1,3-dipolar 
cycloadditions of quinolinium or isoquinolinium methylides to electron deficient dipolarofiles [8], 1,5-electrocyclizations and metal-catalyzed intramolecular $\mathrm{C}-\mathrm{N}$ bond formation of alkynyl pyridines or propargylic pyridines [9].

In the same time, cyclic imides and their $\mathrm{N}$-derivatives are reported to exhibit valuable biological effects including antifungal [10] and antibacterial [11-13] (fig. 1).

In this context, as part of our ongoing research aiming at the design and synthesis of new fused $\mathrm{N}$-heterocyclic compounds with biological activities [14-18], we present here the synthesis, structure and the antimicrobial evaluation of two series of compounds with pyrrolo-pyrrolo[2,1-a]isoquinoline and pyrrolo-pyrrolo[1,2-a]quinoline structures (fig. 1), both series having the end pyrrole cycle as $\mathrm{N}$-substituted cyclic imide.

\section{RESULTS AND DISCUSSION}

We decided to synthesize the pyrrolo-pyrrolo(iso)quinolone skeleton through the $3+2$ cycloaddition of azomethineylide as the 1,3-dipole. This strategy has been used widely to construct complex cyclic systems from relatively simple precursors [19-22].

First step of our synthetic strategy was the synthesis of (iso)quinolinium monoquaternary salts $3-4$ and 6-7 by an $N$-alkylation reaction with reactive halide type 2, of isoquinoline and quinoline, respectively (Scheme 1). Reactions took place at room temperature with good to excellent yields. The structure of the new salts ( 4 and 7 ) was assigned by elemental and spectroscopic analysis: $\mathrm{IR},{ }^{1} \mathrm{H}$ and ${ }^{13} \mathrm{C}$ NMR.<smiles>c1ccc2cnccc2c1</smiles>

1

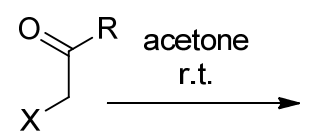

2

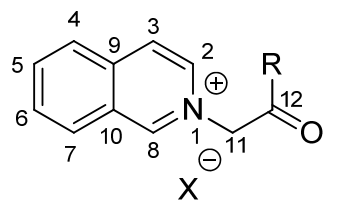

3. $\mathrm{R}=-\mathrm{C}_{6} \mathrm{H}_{4} \mathrm{OMe}(p), \mathrm{X}=\mathrm{Br}$

4. $\mathrm{R}=-\mathrm{NH}_{2}, \mathrm{X}=\mathrm{I}$<smiles>c1ccc2ncccc2c1</smiles>

5

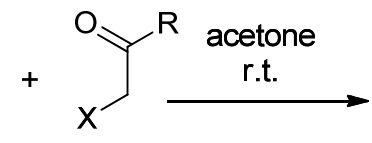

2

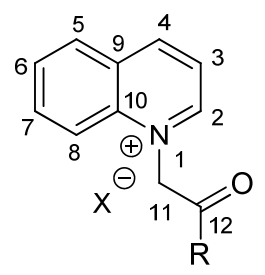

6. $\mathrm{R}=-\mathrm{C}_{6} \mathrm{H}_{4} \mathrm{OMe}(p), \mathrm{X}=\mathrm{Br}$

7. $\mathrm{R}=-\mathrm{NH}_{2}, \mathrm{X}=\mathrm{I}$

Scheme 1. Synthesis of monoquaternary salts $3-4$ and 5-6 
Next, the synthesis of pyrrolo[1,2-a]quinoline and pyrrolo[2,1-a]isoquinoline derivatives took place by $3+2$ cycloaddition of $\mathrm{N}$-ylides generated in situ from salts 3-4 and 6-7 to N-ethyl or N-phenylmaleimide as symmetrically substituted electron deficient alkenes.

Thus, by deprotonation of salts 3-4 and 6-7 under triethylamine treatment, the resonance stabilized $\mathrm{N}$-ylides were in situ generated and reacted with $\mathrm{N}$-ethyl or N-phenylmaleimide giving tetrahydropyrrolo[3',4':3,4]pyrrolo[2,1a]isoquinoline derivatives 9a-b or pyrrolo[3',4':3,4]pyrrolo[2,1-a]isoquinoline derivatives 10a (Scheme 2) and tetrahydropyrrolo[3',4':3,4]pyrrolo[1,2a]quinolines $11 \mathbf{a}-\mathbf{b}$ and $12 \mathbf{a}$, respectively (Scheme 3 ). The cycloaddition reactions with $\mathrm{N}$-ethyl or $\mathrm{N}$-phenylmaleimide occur highly stereoselective, a single isomer being obtained in all cases.

The formation of compound 10a could be explained by an oxidative dehydrogenation of the initial formed corresponding type 9 derivative, leading to the thermodynamically more stable product.

Interesting, the reaction of salts $\mathbf{4}$ and $\mathbf{7}$ with $\mathrm{N}$-phenylmaleimide $\mathbf{8 b}$ proved to be very difficult, complex and inseparable mixtures of compounds being obtained.

The structures of the new compounds $9 a-b, 10 a, 11 a-b$ and $12 a$ were assigned by elemental and spectroscopic analysis (IR, ${ }^{1} \mathrm{H}$ and ${ }^{13} \mathrm{C}-\mathrm{NMR}$ ).
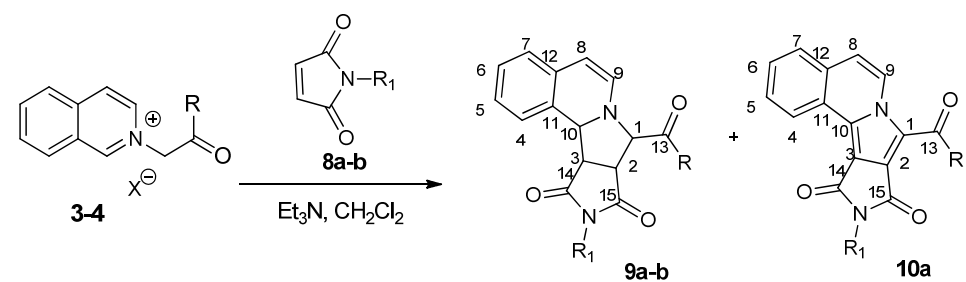

$9 \mathbf{a - b}$

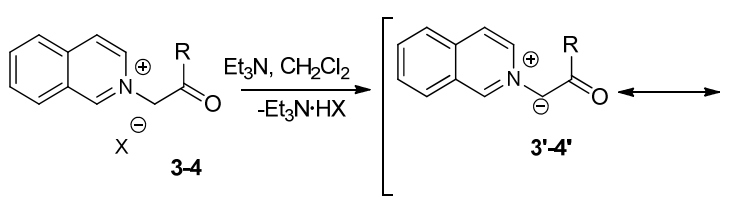

8a. $R_{1}=E t$

8b. $\mathrm{R}_{1}=\mathrm{Ph}$

9a. $\mathrm{R}=-\mathrm{C}_{6} \mathrm{H}_{4} \mathrm{OMe}(p), \mathrm{R}_{1}=\mathrm{Et}$

9b. $\mathrm{R}=-\mathrm{C}_{6} \mathrm{H}_{4} \mathrm{OMe}(p), \mathrm{R}_{1}=\mathrm{Ph}$

10a. $\mathrm{R}=-\mathrm{NH}_{2}, \mathrm{R}_{1}=\mathrm{Et}$

$10 a$
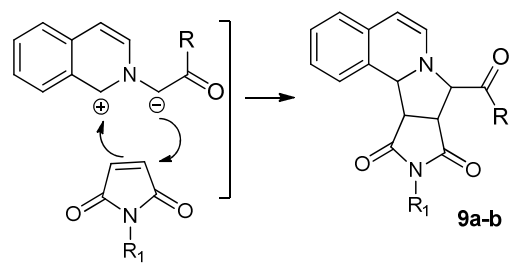

Scheme 2. Synthesis pathway for the tetrahydropyrrolo[3',4':3,4]pyrrolo[2,1a]isoquinoline derivatives $9 a-b$ and the pyrrolo[ $\left.3^{\prime}, 4^{\prime}: 3,4\right]$ pyrrolo[2,1-a]isoquinoline derivative 10a and the proposed reaction mechanism 

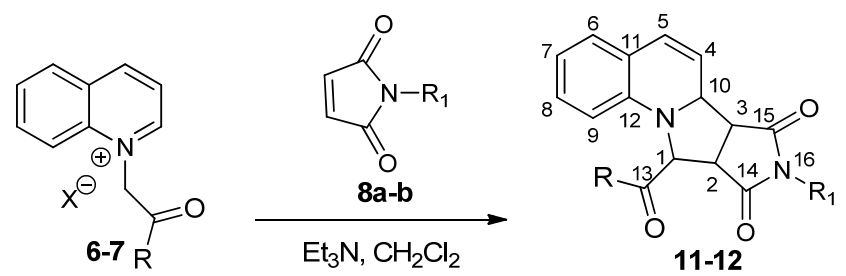

Scheme 3. Synthesis of tetrahydropyrrolo[3',4':3,4]pyrrolo[1,2-a]quinolines $11 \mathrm{a}-\mathrm{b}$ and $12 \mathrm{a}$

The IR spectra of polycyclic compounds type 9a-b show absorption bands between 1774-1761 $\mathrm{cm}^{-1}$ characteristic for in phase stretching vibrations of $\mathrm{C}=\mathrm{O}$ imide groups and between $1696-1692 \mathrm{~cm}^{-1}$ for out of phase vibrations of the same $\mathrm{C}=\mathrm{O}$ imide group in some cases overlapped with ketone or amide carbonyl group absorption. In ${ }^{1} \mathrm{H}$ NMR spectrum of compounds $9 \mathbf{a}-\mathbf{b}$, the signals at $\delta=3.28-3.59 \mathrm{ppm}$ have been assigned to $\mathrm{H} 3$ atom, the most shielded proton of the tetrahydropyrrole cycle. Protons 2 and 10 supply signals at $\delta=3.76-4.15 \mathrm{ppm}$ and $\delta=4.70-4.89 \mathrm{ppm}$, respectively. The signal delivered by $\mathrm{H} 1$ appears as singlet at weak fields $(\delta=5.25-5.51$ $\mathrm{ppm}$ ) because of its electron withdrawing neighbours. The lack of coupling for $\mathrm{H} 1$ proton is a proof for a trans position relative to proton 2. The big values for the coupling constants $J_{2,3}(7.5-8.0 \mathrm{~Hz})$ and $J_{3,10}(8.0 \mathrm{~Hz})$ show us that hydrogen atoms $\mathrm{H} 2, \mathrm{H} 3$ and $\mathrm{H} 10$ lay on the same side of the tetrahydropyrrole ring, but on the opposite side of $\mathrm{H} 1 .{ }^{13} \mathrm{C}-\mathrm{NMR}$ spectra show two different signals for the two carbonyl imide carbon atoms at 174.1-174.6 ppm and 177.6-177.7 ppm respectively, while the carbonyl C13 furnishes signals at 192.0-192.3 ppm.

Compound 10a furnishes a much simpler NMR spectrum with only two signals in the aliphatic region supplied by the ethyl group. Also the singlet signals of two amidic $\mathrm{NH}$ distinguish from the spectrum at $5.62 \mathrm{ppm}$ and 8.71 ppm, respectively.

In ${ }^{1} \mathrm{H}$ NMR of compounds 11-12, there are also four distinct signals in the aliphatic region corresponding to the four hydrogen atoms on the tetrahydropyrrole ring. The signals delivered by $\mathrm{H} 1$ appear as broad singlets, the small coupling $\left(J_{1,2}=1.0 \mathrm{~Hz}\right)$ with $\mathrm{H} 2$ proton being a proof for a trans position relative to proton 1 . On the other hand, the big values for the coupling constants $J_{2,3}(8.0 \mathrm{~Hz})$ and $J_{3,10}(8.0 \mathrm{~Hz})$ show us that hydrogen atoms $\mathrm{H} 2$, $\mathrm{H} 3$ and $\mathrm{H} 10$ lay on the same side of the pyrrole ring, but on the opposite side of $\mathrm{H} 1$. 
The above-mentioned conclusions for compounds type 11-12 were fully sustained by the structure obtained by single-crystal X-ray diffraction. Thus, the X-ray crystal structure of compound 11a, is depicted in Figure 2.

Concerning the space disposal of the four fused rings we may notice that the end pyrrole cycle (former $\mathrm{N}$-ethylmaleimide) is almost perpendicular to the quinoline plane. Full information concerning X-ray structure could be found in the Cambridge Crystallographic Data Centre (CCDC 1936035).

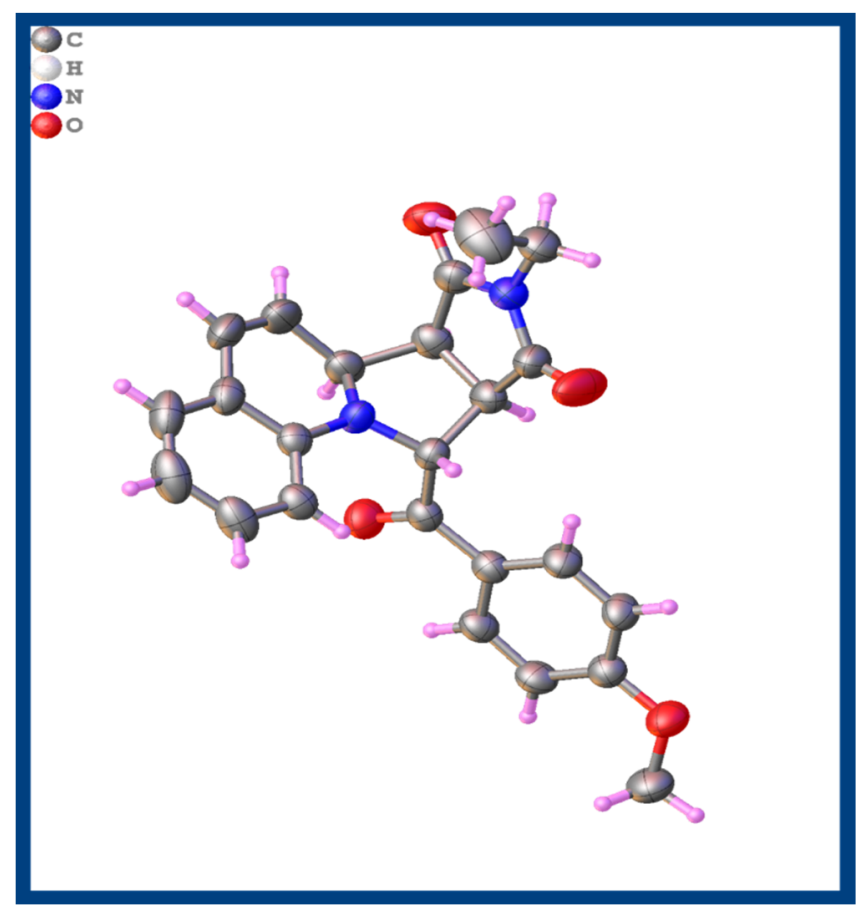

Figure 2. X-ray crystal structure of compound 11a with thermal ellipsoids at $50 \%$ probability level

A perspective view of the 11a compound is shown in Figure 3. From the analysis of molecules packing we can conclude that the crystals are stabilized mainly by rather weak $\mathrm{H}$-bonds $(\mathrm{H}-\mathrm{O}$ length $2.43 \AA$, C-O length $3.17 \AA$ and $\mathrm{C}-\mathrm{H} \cdots \mathrm{O}$ angle $136.4^{\circ}$ ). Also, we noticed the absence of $\pi-\pi$ interactions which is probably a consequence of the nonplanar character of those molecules. Despite those facts the crystal structure is rather compact, the voids size being too small to accommodate solvent molecules. 


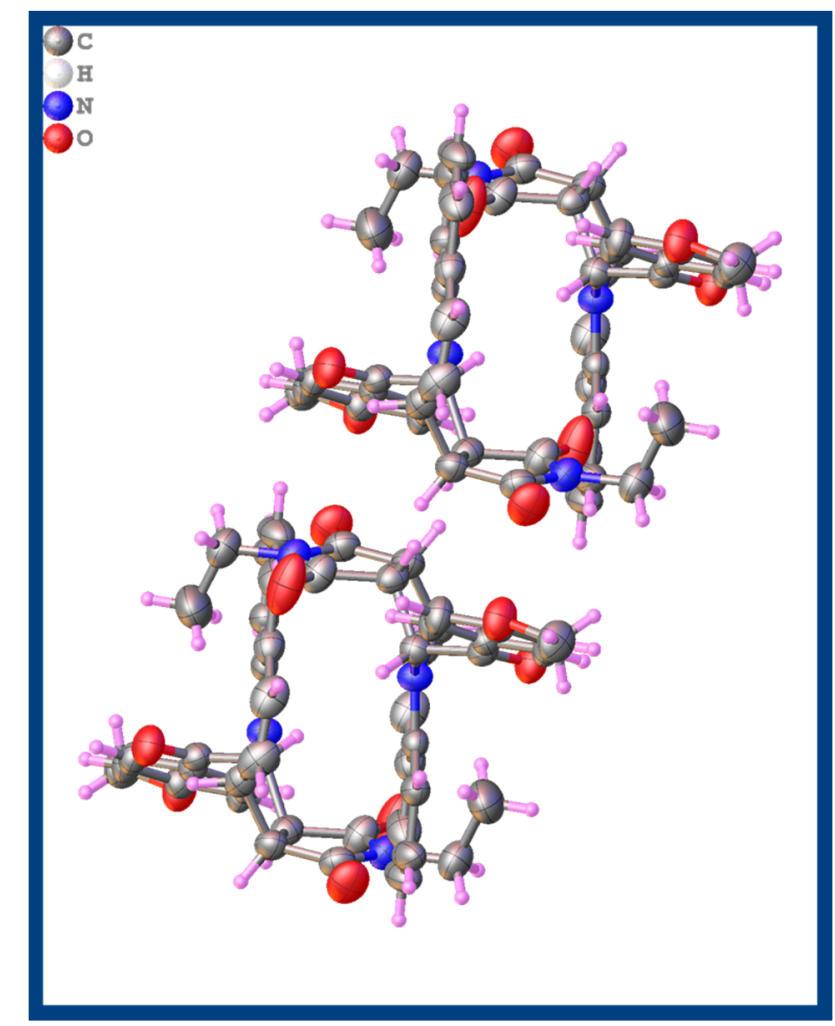

Figure 3. Packing (view along the $c$ axis) of the molecules in the case of compound 11a

\section{Antimicrobial activity}

Our group has previously reported several 1,10-phenanthrolinium monoquaternary salts [23] possessing antibacterial and antifungal activities. Thus, we decided to evaluate the antimicrobial activity for all synthesized compounds, including isoquinolinium salts 3-4 and quinolinium salts 6-7 (excepting salt 7 whose antibacterial evaluation was already reported. [24]. The antimicrobial activity of compounds 3, 4, 6, 7, 9-10 and 11-12 was measured by the agar disk diffusion method [25] which consists of addition of the sterile disks containing the compounds on the culture medium preinoculated with the microbial suspension, and measuring of the clear zone caused by the growth inhibition around the disks after $24 \mathrm{~h}$ of incubation. Unfortunately, all tested compounds showed no antimicrobial activity against all the reference strains. 


\section{CONCLUSIONS}

The synthesis of new fused polycyclic indolizines derived from quinoline and isoquinoline is presented. The strategy adopted for construction of new pyrrolo[1,2-a]quinoline and pyrrolo[2,1-a]isoquinoline derivatives, including a typical Huisgen [3+2] dipolar cycloaddition of the corresponding isoquinolinium and quinolinium $\mathrm{N}$-methylides to $\mathrm{N}$-ethyl and $\mathrm{N}$-phenylmaleimide proved to be straightforward and efficient. The cycloaddition reactions occur highly stereoselective (a single isomer being obtained). X-ray diffraction of compound 11a proved unambiguously the compounds structure and brings information concerning lattice structure. The antibacterial activity of the synthesized compounds was measured, but all the investigated compounds showed no antimicrobial activity against the three reference strains.

\section{EXPERIMENTAL SECTION}

\section{Chemistry}

Melting points were recorded on an A. KrüssOptronic Melting Point Meter KSPI and are uncorrected. Proton and carbon nuclear magnetic resonance $\left(\delta_{\mathrm{H}}, \delta_{\mathrm{C}}\right)$ spectra were recorded on a Bruker Avance III $(500 \mathrm{MHz})$. All chemical shifts are quoted on the $\delta$-scale in ppm. Coupling constants are given in Hz. IR spectra were recorded on a FTIR Shimadzu or Jasco 660 plus FTIR spectrophotometer. Thin layer chromatography (TLC) was carried out on Merck silica gel $60 \mathrm{~F}_{254}$ plates. Column chromatography was carried out on silica gel (Roth $60,0.04-0.063 \mathrm{~mm}$ ). Visualisation of the plates was achieved using a UV lamp $\left(\lambda_{\max }=254\right.$ or $\left.365 \mathrm{~nm}\right)$. All commercially available products were used without further purification unless otherwise specified.

\section{General procedure for synthesis of isoquinolinium and quinolinium salts 3 and 5}

$1 \mathrm{mmol}$ of quinoline or isoquinoline was dissolved in $5 \mathrm{~mL}$ acetone. Then $1.1 \mathrm{mmol}$ of reactive halide was added and the resulted mixture was stirred at room temperature for 24 hours. The formed precipitate was filtered and washed with acetone to give the desired product. 
SYNTHESIS AND ANTIBACTERIAL EVALUATION OF NEW PYRROLO[3', $\left.4^{\prime}: 3,4\right]$ PYRROLO[1,2-a] QUINOLINE AND PYRROLO[3',4':3,4]PYRROLO[2,1-a]ISOQUINOLINE DERIVATIVES

\section{General procedure for synthesis of isoquinoline and quinoline derivatives 7 and 8}

The cycloimmonium salt ( $1 \mathrm{mmol}, 1$ equiv.) and dipolarophile (NEMI or $\mathrm{NFMI}),(1.1 \mathrm{mmol}, 1.1$ equiv), were added to $5 \mathrm{~mL}$ of dichloromethane and the obtained suspension was stirred at room temperature (rt) under $\mathrm{N}_{2}$ atmosphere. Triethylamine (TEA) ( 3 mmol, 3 equiv.) was added drop-wise over 1 hour (magnetic stirring) and the resulting mixture was then stirred for 24 hours at rt. Methanol $(5 \mathrm{~mL})$ was added ant the resulting mixture is kept for 24 hours without stirring. The formed precipitate was collected by filtration to give a solid which was washed with methanol. The product was crystallized from dichloromethane/methanol $(1 / 1, v / v)$.

\section{Spectral data}

2-(2-(4-methoxyphenyl)-2-oxoethyl)isoquinolin-2-ium bromide (3). Grey solid, $n=95 \%$, m.p. $=215-217^{\circ} \mathrm{C}, \mathrm{IR}\left(\mathrm{KBr}, \mathrm{cm}^{-1}\right): 3009,2947,1684$, 1604, 1237, 1162, 974. ${ }^{1} \mathrm{H}-\mathrm{NMR}$ (DMSO-d, $500 \mathrm{MHz}$ ): $\delta=3.91$ (s, 3H, OMe), $6.61\left(\mathrm{~s}, 2 \mathrm{H}, \mathrm{H}_{11}\right), 7.20(\mathrm{~d}, \mathrm{~J}=8.0 \mathrm{~Hz}, 2 \mathrm{H}, 2 \mathrm{xh}-\mathrm{H}$ ), 8.09-8.13 (overlapped signals , $\left.3 \mathrm{H}, \mathrm{H}_{6}, 2 \times \mathrm{Ph}-\mathrm{H}\right), 8.33\left(\mathrm{t}, J=7.5 \mathrm{~Hz}, 1 \mathrm{H}, \mathrm{H}_{5}\right), 8.42(\mathrm{~d}, J=8.5 \mathrm{~Hz}$, $\left.1 \mathrm{H}, \mathrm{H}_{4}\right), 8.55\left(\mathrm{~d}, J=8.0 \mathrm{~Hz}, 1 \mathrm{H}, \mathrm{H}_{7}\right), 8.68\left(\mathrm{~d}, J=7.0 \mathrm{~Hz}, 1 \mathrm{H}, \mathrm{H}_{3}\right), 8.75(\mathrm{~d}, J=$ $6.5 \mathrm{~Hz}, 1 \mathrm{H}, \mathrm{H}_{2}$ ), 10.05 (s, 1H, $\mathrm{H}_{8}$ ). ${ }^{13} \mathrm{C}-\mathrm{NMR}$ (DMSO-d $6,125 \mathrm{MHz}$ ): $\delta=55.9$ (OMe), 65.8 $\mathrm{C}_{11}, 114.5(2 \times \mathrm{CH}-\mathrm{Ph}), 125.4 \mathrm{C}_{3}, 126.4 \mathrm{Cq}-\mathrm{Ph}, 126.9 \mathrm{C}_{10}, 127.4$ $\mathrm{C}_{4}, 130.6 \mathrm{C}_{7}, 130.8(2 \times \mathrm{CH}-\mathrm{Ph}), 131.4 \mathrm{C}_{6}, 136.4 \mathrm{C}_{2}, 137.2 \mathrm{C}_{5}, 137.4 \mathrm{C}_{9}$, $151.7 \mathrm{C}_{8}, 164.4 \mathrm{Cq}-\mathrm{Ph}, 189.2 \mathrm{C}_{12}$.

2-(2-amino-2-oxoethyl)isoquinolin-2-ium iodide (4). Yellow solid, $\eta=97 \%$, m.p. $=199-201^{\circ} \mathrm{C}, \mathrm{IR}\left(\mathrm{KBr}, \mathrm{cm}^{-1}\right): 3334,3147,2927,1691,1649$, 1393, 1292 823. ${ }^{1} \mathrm{H}-\mathrm{NMR}$ (DMSO-d $6,500 \mathrm{MHz}$ ): $\delta=5.53$ (s, 2H, $\left.\mathrm{H}_{11}\right), 7.77$ (s, $1 \mathrm{H}, \mathrm{NH}$ ), 8.10 (as, $2 \mathrm{H}, \mathrm{H}_{6}, \mathrm{NH}$ ), 8.30-8.37 (overlapped signals, $2 \mathrm{H}_{1} \mathrm{H}_{5}, \mathrm{H}_{4}$ ), 8.52-8.70 (overlapped signals, $3 \mathrm{H}, \mathrm{H}_{2}, \mathrm{H}_{3}, \mathrm{H}_{7}$ ), 10.01 (s, $1 \mathrm{H}, \mathrm{H}_{8}$ ). ${ }^{13} \mathrm{C}-\mathrm{NMR}$ (DMSO-d, $125 \mathrm{MHz}$ ): $\delta=61.5 \mathrm{C}_{11}, 125.1 \mathrm{C}_{3}, 126.7 \mathrm{C}_{10}, 127.3 \mathrm{C}_{4}, 130.5 \mathrm{C}_{7}$, $131.3 \mathrm{C}_{6}, 136.4 \mathrm{C}_{2}, 137.1 \mathrm{C}_{9}, 137.3 \mathrm{C}_{5}, 151.6 \mathrm{C}_{8}, 166.2 \mathrm{C}_{12 .}$

1-(2-(4-methoxyphenyl)-2-oxoethyl)quinolin-1-ium bromide (6). Grey solid, $\eta=89 \%$, m.p. $=221-223^{\circ} \mathrm{C}$; IR $\left(\mathrm{KBr}, \mathrm{cm}^{-1}\right): 3024,2956,1673$, 1598, 1519, 1359, 1240, 1175. ${ }^{1} \mathrm{H}-\mathrm{NMR}$ (DMSO-d, $500 \mathrm{MHz}$ ): $\delta=3.92$ (s, $3 \mathrm{H}, \mathrm{OMe}), 7.00\left(\mathrm{~s}, 2 \mathrm{H}, \mathrm{H}_{11}\right), 7.22(\mathrm{~d}, J=8.5 \mathrm{~Hz}, 2 \mathrm{H}, 2 \times \mathrm{Ph}-\mathrm{H}), 8.13(\mathrm{~d}, J=$ $8.5 \mathrm{~Hz}, 2 \mathrm{H}, 2 \times \mathrm{Ph}-\mathrm{H}), 8.06\left(\mathrm{t}, J=7.5 \mathrm{~Hz}, \mathrm{H}_{6}\right), 1 \mathrm{H}, 8.22\left(\mathrm{t}, J=7.5 \mathrm{~Hz}, 1 \mathrm{H}, \mathrm{H}_{7}\right)$, 8.33 (dd, $\left.J=8.5 ; 5.5 \mathrm{~Hz}, 1 \mathrm{H}, \mathrm{H}_{3}\right), 8.40$ (d, $\left.J=8.5 \mathrm{~Hz}, 1 \mathrm{H}, \mathrm{H}_{5}\right), 8.55$ (d, J= 8.5 $\left.\mathrm{Hz}, 1 \mathrm{H}, \mathrm{H}_{8}\right), 9.45\left(\mathrm{~d}, J=8.5 \mathrm{~Hz}, 1 \mathrm{H}, \mathrm{H}_{4}\right), 9.55\left(\mathrm{~d}, J=5.5 \mathrm{~Hz}, 1 \mathrm{H}, \mathrm{H}_{2}\right) \cdot{ }^{13} \mathrm{C}-\mathrm{NMR}$ (DMSO-d $6,125 \mathrm{MHz}): \delta=55.9(\mathrm{OMe}), 62.9 \mathrm{C}_{11}, 114.4(2 \times \mathrm{CH}-\mathrm{Ph}), 119.1 \mathrm{C}_{5}$, $122.2 \mathrm{C}_{3}, 126.4 \mathrm{Cq}-\mathrm{Ph}, 129.4 \mathrm{C}_{9}, 130.0 \mathrm{C}_{6}, 130.6 \mathrm{C}_{8}, 131.2(2 \times \mathrm{CH}-\mathrm{Ph})$, $135.9 \mathrm{C}_{7}, 138.6 \mathrm{C}_{10}, 148.5 \mathrm{C}_{4}, 151.0 \mathrm{C}_{2}, 164.5 \mathrm{Cq}-\mathrm{Ph}, 189.0 \mathrm{C}_{12}$. 
CRISTINA M. AL MATARNEH, MONICA C. SARDARU, MIRCEA O. APOSTU, IRINA ROSCA, CATALINA I. CIOBANU, IONEL I. MANGALAGIU, RAMONA DANAC

1-(2-amino-2-oxoethyl)quinolin-1-ium iodide (7). Yellow solid, $\eta=68 \%$, m.p. $=236-239^{\circ} \mathrm{C}, \mathrm{IR}\left(\mathrm{KBr}, \mathrm{cm}^{-1}\right): 3361,3154,2980,1678,1620,1523,1356$, 778. ${ }^{1} \mathrm{H}-\mathrm{NMR}\left(\mathrm{DMSO}-\mathrm{d}_{6}, 500 \mathrm{MHz}\right): \delta=5.85\left(\mathrm{~s}, 2 \mathrm{H}, \mathrm{H}_{11}\right), 7.84(\mathrm{~s}, 1 \mathrm{H}, \mathrm{NH})$, $8.05\left(\mathrm{dt}, \mathrm{J}=8.0 ; 2.0 \mathrm{~Hz}, 1 \mathrm{H}, \mathrm{H}_{6}\right), 8.17(\mathrm{~s}, 1 \mathrm{H}, \mathrm{NH}), 8.25-8.31\left(\mathrm{~m}, 3 \mathrm{H}, \mathrm{H}_{3}, \mathrm{H}_{7}\right.$, $\left.\mathrm{H}_{5}\right), 8.51\left(\mathrm{~d}, J=8.0 \mathrm{~Hz}, 1 \mathrm{H}, \mathrm{H}_{8}\right), 9.38\left(\mathrm{~d}, J=8.0 \mathrm{~Hz}, 1 \mathrm{H}, \mathrm{H}_{4}\right), 9.52(\mathrm{~d}, J=5.5 \mathrm{~Hz}$, $1 \mathrm{H}, \mathrm{H}_{2}$ ). ${ }^{13} \mathrm{C}-\mathrm{NMR}\left(\mathrm{DMSO}-\mathrm{d}_{6}, 125 \mathrm{MHz}\right.$ ): $\delta=58.8 \mathrm{C}_{11}, 118.4 \mathrm{C}_{5}, 122.0 \mathrm{C}_{3}, 129.3$ $\mathrm{C}_{9}, 129.9 \mathrm{C}_{6}, 130.6 \mathrm{C}_{8}, 135.9 \mathrm{C}_{7}, 138.2 \mathrm{C}_{10}, 148.2 \mathrm{C}_{4}, 151.1 \mathrm{C}_{2}, 165.8 \mathrm{C}_{12}$.

10-ethyl-8-(4-methoxybenzoyl)-8,8a,11a,11b-tetrahydro-9Hpyrrolo[3',4':3,4]pyrrolo[2,1-a]isoquinoline-9,11(10H)-dione (9a). Yellow crystals, $\eta=60 \%$, m.p. $=208-210^{\circ} \mathrm{C}$; IR $(\mathrm{KBr}), \mathrm{v}\left(\mathrm{cm}^{-1}\right): 2978,1774,1696$, 1670, 1600, 1399, 1225. ${ }^{1} \mathrm{H}-\mathrm{NMR}\left(\mathrm{CDCl}_{3}, 500 \mathrm{MHz}\right): \delta=0.90(\mathrm{t}, \mathrm{J}=7.0 \mathrm{~Hz}$, $\left.3 \mathrm{H}, \mathrm{CH}_{2} \mathrm{CH}_{3}\right), 3.25$ (q, J= $\left.7.0 \mathrm{~Hz}, 2 \mathrm{H}, \mathrm{CH}_{2} \mathrm{CH}_{3}\right), 3.28\left(\mathrm{t}, J=8.0 \mathrm{~Hz}, 1 \mathrm{H}, \mathrm{H}_{3}\right)$, $3.73(1 \mathrm{H}, \mathrm{s}, \mathrm{OMe}), 3.76\left(\mathrm{~d}, J=7.5 \mathrm{~Hz}, 1 \mathrm{H}, \mathrm{H}_{2}\right), 4.70\left(\mathrm{~d}, J=8.0 \mathrm{~Hz}, 1 \mathrm{H}, \mathrm{H}_{10}\right)$, $5.16\left(\mathrm{~d}, J=7.5 \mathrm{~Hz}, 1 \mathrm{H}, \mathrm{H}_{8}\right), 5.29\left(\mathrm{~s}, 1 \mathrm{H}, \mathrm{H}_{1}\right), 6.05\left(\mathrm{~d}, J=7.5 \mathrm{~Hz}, 1 \mathrm{H}, \mathrm{H}_{9}\right), 6.77$ (d, $\left.J=7.5 \mathrm{~Hz}, 1 \mathrm{H}, \mathrm{H}_{4}\right), 6.83(\mathrm{~d}, J=8.5 \mathrm{~Hz}, 2 \mathrm{H}, 2 \times \mathrm{Ph}-\mathrm{H}), 7.00$ (as, $2 \mathrm{H}, \mathrm{H}_{5}$, $\mathrm{H}_{6}$ ), 7.03 (dd, $J=7.5$; $4.0 \mathrm{~Hz}, 1 \mathrm{H}, \mathrm{H}_{7}$ ), 7.95 (d, $\left.J=8.5 \mathrm{~Hz}, 2 \mathrm{H}, 2 \times \mathrm{Ph}-\mathrm{H}\right) .{ }^{13} \mathrm{C}-$ $\operatorname{NMR}\left(\mathrm{CDCl}_{3}, 125 \mathrm{MHz}\right): \delta=11.4\left(\mathrm{CH}_{2} \underline{\mathrm{CH}_{3}}\right), 33.8\left(\underline{\mathrm{C}_{2}} \mathrm{CH}_{3}\right), 45.8 \mathrm{C}_{2}, 51.3 \mathrm{C}_{3}$, 55.4 (OMe), $61.7 \mathrm{C}_{10}, 72.1 \mathrm{C}_{1}, 102.5 \mathrm{C}_{8}, 113.8(2 \times \mathrm{CH}-\mathrm{Ph}), 124.2 \mathrm{C}_{4}, 125.7$ $\mathrm{C}_{6}, 125.8 \mathrm{C}_{11}, 126.5 \mathrm{Cq}-\mathrm{Ph}, 127.3 \mathrm{C}_{5}, 128.2 \mathrm{C}_{7}, 130.8 \mathrm{C}_{12}, 131.4(2 \times \mathrm{CH}-$ $\mathrm{Ph}), 134.9 \mathrm{C}_{9}, 163.9 \mathrm{Cq}-\mathrm{Ph}, 174.6 \mathrm{C}_{14}, 177.7 \mathrm{C}_{15}, 192.0 \mathrm{C}_{13}$.

8-(4-methoxybenzoyl)-10-phenyl-8,8a,11a,11b-tetrahydro-9Hpyrrolo[3',4':3,4]pyrrolo[2,1-a]isoquinoline-9,11(10H)-dione (9b). Yellow solid, $\eta=90 \%$, m.p. $=208-210^{\circ} \mathrm{C}$; IR $(\mathrm{KBr}), \mathrm{v}\left(\mathrm{cm}^{-1}\right): 1761,1692,1671,1595$, 1507, 1394, 1240, 1167. ${ }^{1} \mathrm{H}-\mathrm{NMR}\left(\mathrm{CDCl}_{3}, 500 \mathrm{MHz}\right): \delta=3.59$ (t, $J=8.0 \mathrm{~Hz}$, $\left.1 \mathrm{H}, \mathrm{H}_{3}\right), 3.86(1 \mathrm{H}, \mathrm{s}, \mathrm{OMe}), 4.15\left(\mathrm{~d}, J=8.0 \mathrm{~Hz}, 1 \mathrm{H}, \mathrm{H}_{2}\right), 4.89(\mathrm{~d}, J=8.0 \mathrm{~Hz}$, $\left.1 \mathrm{H}, \mathrm{H}_{10}\right), 5.49\left(\mathrm{~d}, J=7.5 \mathrm{~Hz}, 1 \mathrm{H}, \mathrm{H}_{8}\right), 5.51\left(\mathrm{~s}, 1 \mathrm{H}, \mathrm{H}_{1}\right), 6.25(\mathrm{~d}, J=7.5 \mathrm{~Hz}, 1 \mathrm{H}$, $\left.\mathrm{H}_{9}\right), 7.12-7.20\left(\mathrm{~m}, 3 \mathrm{H}, \mathrm{H}_{4}, \mathrm{H}_{6}, \mathrm{H}_{5}\right), 6.96\left(\mathrm{ad}, \mathrm{J}=8.5 \mathrm{~Hz}, 3 \mathrm{H}, 2 \times \mathrm{Ph}-\mathrm{H}, \mathrm{H}_{7}\right)$, 7.09 (d, J= $7.5 \mathrm{~Hz}, 2 \mathrm{H}, 2 \times \mathrm{Ph}-\mathrm{H}), 7.33$ (t, J= $7.5 \mathrm{~Hz}, 1 \mathrm{H}, \mathrm{Ph}-\mathrm{H}), 7.40$ (t, J= $7.5 \mathrm{~Hz}, 2 \mathrm{H}, 2 \times \mathrm{Ph}-\mathrm{H}), 8.09(\mathrm{~d}, J=8.5 \mathrm{~Hz}, 2 \mathrm{H}, 2 \times \mathrm{Ph}-\mathrm{H}) .{ }^{13} \mathrm{C}-\mathrm{NMR}\left(\mathrm{CDCl}_{3}\right.$, $125 \mathrm{MHz}$ ): $\delta=46.5 \mathrm{C}_{2}, 51.9 \mathrm{C}_{3}, 55.7$ (OMe), $62.5 \mathrm{C}_{10}, 73.3 \mathrm{C}_{1}, 103.1 \mathrm{C}_{8}$, $114.2(2 \times \mathrm{CH}-\mathrm{Ph}), 124.8 \mathrm{C}_{7}, 126.0\left(2 \times \mathrm{Ph}-\mathrm{CH}, \mathrm{C}_{11}\right), 126.5 \mathrm{C}_{6}, 126.9 \mathrm{C}_{14}$, 127.8 $\mathrm{C}_{4}, 128.6(\mathrm{Ph}-\mathrm{CH}), 128.9 \mathrm{C}_{5}, 129.2(2 \times \mathrm{Ph}-\mathrm{CH}), 130.9 \mathrm{C}_{12}, 131.8(2 \times$ $\mathrm{CH}-\mathrm{Ph}), 132.1 \mathrm{C}_{\mathrm{g}} \mathrm{Ph}, 135.3 \mathrm{C}_{9}, 164.4 \mathrm{C}_{17}, 174.1 \mathrm{C}_{14}, 177.6 \mathrm{C}_{15}, 192.3 \mathrm{C}_{13}$.

10-ethyl-8-(4-methoxybenzoyl)-9H-pyrrolo[3',4':3,4]pyrrolo[2,1-

a]isoquinoline-9,11(10H)-dione (10a). Brown solid, $\eta=60 \%$, m.p. $=239$ $240^{\circ} \mathrm{C}$. IR $\left(\mathrm{KBr}, \mathrm{cm}^{-1}\right): 3394,3182,1741,1687,1618,1408,1221,1018 .{ }^{1} \mathrm{H}-$ $\operatorname{NMR}\left(\mathrm{CDCl}_{3}, 500 \mathrm{MHz}\right): \delta=1.33\left(\mathrm{t}, \mathrm{J}=7.0 \mathrm{~Hz}, 3 \mathrm{H}, \mathrm{CH}_{2} \mathrm{CH}_{3}\right), 3.79$ (q, $J=7.0$ $\left.\mathrm{Hz}, 2 \mathrm{H}, \mathrm{CH}_{2} \mathrm{CH}_{3}\right), 5.62(\mathrm{~s}, 1 \mathrm{H}, \mathrm{NH}), 7.22\left(\mathrm{~d}, J=7.5 \mathrm{~Hz}, 1 \mathrm{H}, \mathrm{H}_{8}\right), 7.67-7.76$ (overlapped signals, $\left.3 \mathrm{H}, \mathrm{H}_{5}, \mathrm{H}_{6}, \mathrm{H}_{7}\right), 8.71(\mathrm{~s}, 1 \mathrm{H}, \mathrm{NH}), 9.11(\mathrm{~d}, J=7.5 \mathrm{~Hz}, 1 \mathrm{H}$, $\left.\mathrm{H}_{4}\right), 9.64\left(\mathrm{~d}, J=7.5 \mathrm{~Hz}, 1 \mathrm{H}, \mathrm{H}_{9}\right) \cdot{ }^{13} \mathrm{C}-\mathrm{NMR}\left(\mathrm{CDCl}_{3}, 125 \mathrm{MHz}\right): \delta=14.3$ $\left(\mathrm{CH}_{2} \underline{\mathrm{C}} \mathrm{H}_{3}\right), 33.7\left(\mathrm{CH}_{2} \mathrm{CH}_{3}\right), 111.0 \mathrm{C}_{3}, 114.8 \mathrm{C}_{1}, 115.9 \mathrm{C}_{8}, 124.0 \mathrm{C}_{11}, 125.8 \mathrm{C}_{9}$, $126.8 \mathrm{C}_{7}, 127.4 \mathrm{C}_{4}, 129.0 \mathrm{C}_{5}, 129.5 \mathrm{C}_{2}, 130.4 \mathrm{C}_{6}, 131.6 \mathrm{C}_{12}, 151.8 \mathrm{C}_{10}, 161.2$ $\mathrm{C}_{14}, 163.7 \mathrm{C}_{13}, 166.2 \mathrm{C}_{15}$. 
SYNTHESIS AND ANTIBACTERIAL EVALUATION OF NEW PYRROLO[3', 4':3,4]PYRROLO[1,2-a] QUINOLINE AND PYRROLO[3',4':3,4]PYRROLO[2,1-a]ISOQUINOLINE DERIVATIVES

\section{8-ethyl-10-(4-methoxybenzoyl)-6a,6b,9a,10-tetrahydro-7H-pyrrolo} [3',4':3,4]pyrrolo[1,2-a]quinoline-7,9(8H)-dione (11a). Yellow crystals, $\eta=$ $70 \%$, m.p. $=190-192^{\circ} \mathrm{C} ; \mathrm{IR}(\mathrm{KBr}), \mathrm{v}\left(\mathrm{cm}^{-1}\right): 3056,2974 ; 2930,2827,1783,1698$, 1598, 1491, 1446, 1397, 1233, 1166, 759. ${ }^{1} \mathrm{H}-\mathrm{NMR}\left(\mathrm{CDCl}_{3}, 500 \mathrm{MHz}\right): \delta=1.03$ $\left(\mathrm{t}, J=7.0 \mathrm{~Hz}, 3 \mathrm{H}, \mathrm{CH}_{2} \mathrm{CH}_{3}\right), 3.41\left(\mathrm{t}, J=8.0 \mathrm{~Hz}, 1 \mathrm{H}, \mathrm{H}_{3}\right), 3.52(\mathrm{q}, J=7.0 \mathrm{~Hz}, 2 \mathrm{H}$, $\mathrm{C}_{2} \mathrm{CH}_{3}$ ), 3.57 (dd, $\left.J=8.0,1.0 \mathrm{~Hz}, 1 \mathrm{H}, \mathrm{H}_{2}\right), 3.91(1 \mathrm{H}, \mathrm{s}, \mathrm{OMe}), 5.20$ (td, $J=8.0$, $\left.2.0 \mathrm{~Hz}, 1 \mathrm{H}, \mathrm{H}_{10}\right), 5.63\left(\mathrm{~s}, 1 \mathrm{H}, \mathrm{H}_{1}\right), 6.05$ (dd, $\left.J=10.0,2.0 \mathrm{~Hz}, 1 \mathrm{H}, \mathrm{H}_{4}\right), 6.18(\mathrm{~d}$, $\left.J=8.0 \mathrm{~Hz}, 1 \mathrm{H}, \mathrm{H}_{9}\right), 6.40\left(\mathrm{dd}, J=10.0,2.0 \mathrm{~Hz}, 1 \mathrm{H}, \mathrm{H}_{5}\right), 6.65(\mathrm{t}, J=7.5 \mathrm{~Hz}, 1 \mathrm{H}$, $\left.\mathrm{H}_{7}\right), 6.92\left(\mathrm{~d}, J=7.0 \mathrm{~Hz}, 1 \mathrm{H}, \mathrm{H}_{6}\right), 6.96\left(\mathrm{t}, J=8.5 \mathrm{~Hz}, 2 \mathrm{H}, \mathrm{H}_{8}\right), 7.04(\mathrm{~d}, J=8.5 \mathrm{~Hz}$, $2 \mathrm{H}, 2 \times \mathrm{Ph}-\mathrm{H}), 8.28$ (d, J= $8.5 \mathrm{~Hz}, 2 \mathrm{H}, 2 \times \mathrm{Ph}-\mathrm{H}) \cdot{ }^{13} \mathrm{C}-\mathrm{NMR}\left(\mathrm{CDCl}_{3}, 125 \mathrm{MHz}\right)$ : $\delta=12.9\left(\mathrm{CH}_{2} \underline{\mathrm{C}} \mathrm{H}_{3}\right), 34.5\left(\mathrm{C}_{2} \mathrm{CH}_{3}\right), 47.0 \mathrm{C}_{2}, 48.2 \mathrm{C}_{3}, 55.7(\mathrm{OMe}), 60.7 \mathrm{C}_{10}$, $63.8 \mathrm{C}_{1}, 110.7 \mathrm{C}_{9}, 114.5(2 \times \mathrm{CH}-\mathrm{Ph}), 118.8 \mathrm{C}_{7}, 121.1 \mathrm{C}_{4}, 122.2 \mathrm{C}_{11}, 126.6$ $\mathrm{Cq}-\mathrm{Ph}, 126.7 \mathrm{C}_{5}, 127.5 \mathrm{C}_{6}, 129.2 \mathrm{C}_{8}, 131.6(2 \times \mathrm{CH}-\mathrm{Ph}), 141.8 \mathrm{C}_{12}, 164.5 \mathrm{Cq}-$ $\mathrm{Ph}, 175.7 \mathrm{C}_{14}, 177.6 \mathrm{C}_{15}, 194.2 \mathrm{C}_{13}$.

10-(4-methoxybenzoyl)-8-phenyl-6a,6b,9a,10-tetrahydro-7H-pyrrolo [3',4':3,4]pyrrolo[1,2-a]quinoline-7,9(8H)-dione (11b). Yellow crystals, $n=$ $80 \%$, m. p. $=198-199^{\circ} \mathrm{C} ; \mathrm{IR}(\mathrm{KBr}), \mathrm{v}\left(\mathrm{cm}^{-1}\right): 3042,2962,2909,1779,1715,1676$, 1599, 1489, 1379, 1172, 746. ${ }^{1} \mathrm{H}-\mathrm{NMR}\left(\mathrm{CDCl}_{3}, 500 \mathrm{MHz}\right): \delta=3.59(\mathrm{t}, J=8.0$ $\left.\mathrm{Hz}, 1 \mathrm{H}, \mathrm{H}_{3}\right), 3.82\left(\mathrm{~d}, J=8.0 \mathrm{~Hz}, 1 \mathrm{H}, \mathrm{H}_{2}\right), 3.91(1 \mathrm{H}, \mathrm{s}, \mathrm{OMe}), 5.24$ (td, $J=8.0$, $2.0 \mathrm{~Hz}, 1 \mathrm{H}, \mathrm{H}_{10}$ ), $5.80\left(\mathrm{~s}, 1 \mathrm{H}, \mathrm{H}_{1}\right), 6.09$ (dd, $\left.J=10.0,2.5 \mathrm{~Hz}, 1 \mathrm{H}, \mathrm{H}_{4}\right), 6.31$ (d, $\left.J=8.0 \mathrm{~Hz}, 1 \mathrm{H}, \mathrm{H}_{9}\right), 6.46\left(\mathrm{dd}, J=10.0,2.0 \mathrm{~Hz}, 1 \mathrm{H}, \mathrm{H}_{5}\right), 6.71(\mathrm{t}, J=7.5 \mathrm{~Hz}, 1 \mathrm{H}$, $\left.\mathrm{H}_{7}\right), 6.97\left(\mathrm{~d}, J=7.5 \mathrm{~Hz}, 1 \mathrm{H}, \mathrm{H}_{6}\right), 7.01\left(\mathrm{t}, J=8.0 \mathrm{~Hz}, 2 \mathrm{H}, \mathrm{H}_{8}\right), 7.03(\mathrm{~d}, J=8.5 \mathrm{~Hz}$, $2 \mathrm{H}, 2 \times \mathrm{Ph}-\mathrm{H}$ ), 7.10 (d, J= 7.5 Hz, 2H, $2 \times \mathrm{Ph}-\mathrm{H}), 7.35-7.43(\mathrm{~m}, 3 \mathrm{H}, 3 \times \mathrm{Ph}-\mathrm{H})$, $8.29(\mathrm{~d}, \mathrm{~J}=8.5 \mathrm{~Hz}, 2 \mathrm{H}, 2 \times \mathrm{Ph}-\mathrm{H}) .{ }^{13} \mathrm{C}-\mathrm{NMR}\left(\mathrm{CDCl}_{3}, 125 \mathrm{MHz}\right): \delta=47.1 \mathrm{C}_{2}$, $48.7 \mathrm{C}_{3}, 55.8(\mathrm{OMe}), 61.0 \mathrm{C}_{10}, 64.6 \mathrm{C}_{1}, 110.9 \mathrm{C}_{9}, 114.5(2 \times \mathrm{CH}-\mathrm{Ph}), 119.1 \mathrm{C}_{7}$, $121.0 \mathrm{C}_{4}, 122.2 \mathrm{C}_{11}, 126.5(2 \times \mathrm{Ph}-\mathrm{CH}), 126.6 \mathrm{Cq}-\mathrm{Ph}, 127.0 \mathrm{C}_{5}, 127.7 \mathrm{C}_{6}$, 129.0 (CH-Ph), 129.3 (2 x Ph-CH), $129.4 \mathrm{C}_{8}, 131.6$ ( $\left.\mathrm{C}_{15}, \mathrm{C}_{19}, \mathrm{Ph}-\mathrm{Cq}\right), 141.7$ $\mathrm{C}_{12}, 164.6 \mathrm{Cq}-\mathrm{Ph}_{17}, 175.0 \mathrm{C}_{14}, 177.0 \mathrm{C}_{15}, 194.1 \mathrm{C}_{13}$.

8-ethyl-7,9-dioxo-6b,7,8,9,9a,10-hexahydro-6aH-pyrrolo[3',4':3,4] pyrrolo[1,2-a]quinoline-10-carboxamide (12a). Brown solid, $\eta=70 \%$, m.p. $=213-217^{\circ} \mathrm{C} . \mathrm{IR}\left(\mathrm{KBr}, \mathrm{cm}^{-1}\right): 3401,3259,2984,1762,1690,1600,1406$, 1218, 1125, 759, 614. ${ }^{1} \mathrm{H}-\mathrm{NMR}$ (DMSO-d6, $500 \mathrm{MHz}$ ): $\delta=0.81$ (t, $J=7.0 \mathrm{~Hz}$, $3 \mathrm{H}, \mathrm{CH}_{2} \mathrm{CH}_{3}$ ), 3.3 (q, J= $7.0 \mathrm{~Hz}, 2 \mathrm{H}, \mathrm{CH}_{2} \mathrm{CH}_{3}$ ), 3.55 (overlapped signals, $2 \mathrm{H}$, $\left.\mathrm{H}_{3}, \mathrm{H}_{2}\right), 4.59\left(\mathrm{~s}, 1 \mathrm{H}, \mathrm{H}_{1}\right), 5.11\left(\mathrm{~m}, 1 \mathrm{H}, \mathrm{H}_{10}\right), 5.88\left(\mathrm{dd}, \mathrm{J}=10.0,2.5 \mathrm{~Hz}, 1 \mathrm{H}, \mathrm{H}_{4}\right)$, $6.34\left(\mathrm{dd}, J=10.0,2.0 \mathrm{~Hz}, 1 \mathrm{H}, \mathrm{H}_{5}\right), 6.52\left(\mathrm{~d}, J=8.0 \mathrm{~Hz}, 1 \mathrm{H}, \mathrm{H}_{9}\right), 6.58(\mathrm{t}, J=7.0$ $\left.\mathrm{Hz}, 1 \mathrm{H}, \mathrm{H}_{7}\right), 6.89\left(\mathrm{dd}, J=7.5 ; 1.0 \mathrm{~Hz}, 1 \mathrm{H}, \mathrm{H}_{6}\right), 6.98(\mathrm{dt}, J=8.5 ; 1.0 \mathrm{~Hz}, 1 \mathrm{H}$, $\left.\mathrm{H}_{8}\right), 7.39(\mathrm{~s}, 1 \mathrm{H}, \mathrm{NH}), 7.92(\mathrm{~s}, 1 \mathrm{H}, \mathrm{NH}) \cdot{ }^{13} \mathrm{C}-\mathrm{NMR}\left(\mathrm{CDCl}_{3}, 125 \mathrm{MHz}\right): \delta=$ 12.4 $\left(\mathrm{CH}_{2} \underline{\mathrm{C}} \mathrm{H}_{3}\right), 33.2\left(\underline{\mathrm{CH}_{2}} \mathrm{CH}_{3}\right), 47.3 \mathrm{C}_{2}, 48.5 \mathrm{C} 3,59.4 \mathrm{C}_{10}, 62.0 \mathrm{C}_{1}, 110.5 \mathrm{C}_{9}$, $117.8 \mathrm{C}_{7}, 121.1 \mathrm{C}_{11}, 121.6 \mathrm{C}_{4}, 125.4 \mathrm{C}_{5}, 126.9 \mathrm{C}_{6}, 128.9 \mathrm{C}_{8}, 142.1 \mathrm{C}_{12}, 172.1$ $\mathrm{C}_{13}, 175.9 \mathrm{C}_{14}, 177.4 \mathrm{C}_{15}$. 
CRISTINA M. AL MATARNEH, MONICA C. SARDARU, MIRCEA O. APOSTU, IRINA ROSCA, CATALINA I. CIOBANU, IONEL I. MANGALAGIU, RAMONA DANAC

\section{X-Ray Crystallography}

Crystallographic measurements for compound $\mathrm{C}_{24} \mathrm{H}_{22} \mathrm{~N}_{2} \mathrm{O}_{4}$ were carried out on a SuperNova, Dual, Cu at zero, Eos diffractometer. The crystal was kept at $294 \mathrm{~K}$ during data collection. The unit cell determination and data integration were carried out using the CrysAlisPro package of Oxford Diffraction. Using Olex2 [26], the structure was solved with the Superflip [27] structure solution program using Charge Flipping and refined with the ShelXL [28] refinement package using Least Squares minimisation.

Table 1. Crystal data and structure refinement for $11 \mathrm{a}$

\begin{tabular}{|c|c|}
\hline Identification code & $11 a$ \\
\hline Empirical formula & $\mathrm{C}_{24} \mathrm{H}_{22} \mathrm{~N}_{2} \mathrm{O}_{4}$ \\
\hline Formula weight & 402.45 \\
\hline Temperature/K & 294 \\
\hline Crystal system & triclinic \\
\hline Space group & P-1 \\
\hline $\mathrm{a} / \AA$ & $8.9438(5)$ \\
\hline $\mathrm{b} / \AA \AA$ & $9.4257(4)$ \\
\hline $\mathrm{c} / \AA \AA$ & $13.1464(6)$ \\
\hline $\bar{\alpha} /^{\circ}$ & $92.456(3)$ \\
\hline$\beta /^{\circ}$ & $107.620(4)$ \\
\hline $\mathrm{y} /{ }^{\circ}$ & $106.925(4)$ \\
\hline Volume/ $/ \AA^{3}$ & 1000.05(9) \\
\hline Z & 2 \\
\hline$\rho_{\text {calc }} \mathrm{mg} / \mathrm{mm}^{3}$ & 1.3364 \\
\hline $\mathrm{m} / \mathrm{mm}^{-1}$ & 0.747 \\
\hline $\mathrm{F}(000)$ & 425.4 \\
\hline Crystal size $/ \mathrm{mm}^{3}$ & $0.3200 \times 0.2800 \times 0.2200$ \\
\hline Radiation & Cu Ka $(\lambda=1.54184)$ \\
\hline $2 \Theta$ range for data collection & 7.12 to $140.88^{\circ}$ \\
\hline Index ranges & $-10 \leq h \leq 10,-11 \leq k \leq 9,-16 \leq \mathrm{I} \leq 15$ \\
\hline Reflections collected & 6411 \\
\hline Independent reflections & $3732[R($ int $)=0.0124]$ \\
\hline Data/restraints/parameters & $3732 / 0 / 272$ \\
\hline Goodness-of-fit on $F^{2}$ & 1.059 \\
\hline Final $R$ indexes $[\mid>=2 \sigma(I)]$ & $\mathrm{R}_{1}=0.0426, \mathrm{wR}_{2}=\mathrm{N} / \mathrm{A}$ \\
\hline Final $\mathrm{R}$ indexes [all data] & $\mathrm{R}_{1}=0.0456, \mathrm{wR} \mathrm{R}_{2}=0.1136$ \\
\hline Largest diff. peak/hole / e $\AA^{-3}$ & $0.17 /-0.20$ \\
\hline
\end{tabular}


The main crystallographic data together with refinement details are summarized in Table 1. CCDC 1936035 contains the full crystallographic data. Those data can be obtained free of charge from the Cambridge Crystallographic Data Centre via www.ccdc.cam.ac.uk/data_request/cif.

\section{Antimicrobial activity}

The antimicrobial activity was determined by disk diffusion assay against three different reference strains: Staphylococcus aureus ATCC25923, Escherichia coli ATCC25922 and Candida albicans ATCC10231. All microorganisms were stored at $-80^{\circ} \mathrm{C}$ in $20 \%$ glycerol. The bacterial strains (S. aureus and E. coli) were refreshed in Mueller-Hinton broth at $36 \pm 1^{\circ} \mathrm{C}$ and the yeast strain (C. albicans) was refreshed on Sabouraud dextrose broth at $36 \pm 1^{\circ} \mathrm{C}$. Microbial suspensions were prepared with these cultures in sterile solution to obtain turbidity optically comparable to that of 0.5 McFarland

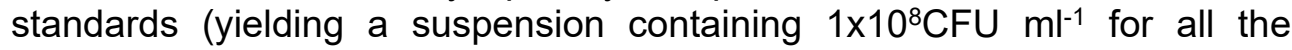
microorganisms).

Volumes of $0.4 \mathrm{ml}$ from each inoculum were spread onto MuellerHinton agar and Sabouraud dextrose agar and all the tested samples were added after the medium surface dried. The sterilized paper discs $(6 \mathrm{~mm})$ were placed on the plate and an aliquot $(100 \mu \mathrm{l})$ of the tested compounds (concentration $10 \mathrm{mg} / \mathrm{ml}$, dissolved in DMSO) was added on the paper discs. To evaluate the antimicrobial properties, the growth inhibition was measured under standard conditions after 24 hours of incubation at $36 \pm 1{ }^{\circ} \mathrm{C}$. After incubation, the diameters of inhibition zones were measured by using Image J software.

\section{ACKNOWLEDGMENTS}

We thank the POSCCE-O 2.2.1, SMIS-CSNR 13984-901, Project no. 257/28.09.2010, CERNESIM, for the NMR and XRD experiments.

\section{REFERENCES}

1. I.M. Gould; Int. J. Antimicrob. Ag., 2008, 32S, S2-S9.

2. G L. French; Int. J. Antimicrob.Ag., 2010, 36S3, S3-S7.

3. M.G. Ferlin; C. Marzano; L. Dalla Via; A. Chilin; G. Zagotto; A. Guiotto; S. Moro; Bioorg. Med. Chem., 2005, 13, 4733-4739. 
CRISTINA M. AL MATARNEH, MONICA C. SARDARU, MIRCEA O. APOSTU, IRINA ROSCA, CATALINA I. CIOBANU, IONEL I. MANGALAGIU, RAMONA DANAC

4. J. Krishnan; B. Vedhanarayanan; B. S. Sasidhar; S. Varughese; V. Nai; Chem. Asian J., 2017, 12, $623-627$.

5. J.W. Daly; B. Witkop; T. Tokuyama; T. Nishikawa; I.L. Karle; Helv. Chim. Acta, 1977, 60, 1128-1140.

6. H. Ishibashi; S. Harada; K. Sato; M. Ikeda; S. Akai; Y. Tamura; V.U. Ahmad; A. Rahman; T. Rasheed; H. Rehman; Heterocycles ,1987, 26, 1251.

7. D.S. Allgäuer; H. Mayr; Eur. J. Org. Chem., 2014, 14, 2956-2963.

8. Y. Liu; Y. Zhang; Y.-M.Shen; H.-W. Hu; J.-H.Xu; Org. Biomol. Chem., 2010, 8, 2449-2456.

9. J. An; Q.-Q. Yang; Q. Wang; W.-J. Xiao; Tetrahedron Lett., 2013, 54, 38343837.

10. R.S. Dhivare; S.S. Rajput; Int. J. Chem. Tech. Res., 2016, 9(3), 325-331.

11. A.M. Al- Azzawi; K.K.H. Al-Obiadi; Int. J. Res. Pharm. \&Chem., 2016, 6(1), 1-8.

12. C. Jin; R. Alenazy; Y. Wang; R. Mowla; Y. Qin; J. Quan; E. Tan; N.D. Modi; X. Gu; S.W. Polyak; H. Venter; S. Ma; Bioorg. Med. Chem. Let., 2019, 19(7), 882889.

13. T.H. Largani; G. Imanzadeh; S. Zahri; N.N. Pesyan; E. Şahin; Green Chem. Lett. Rev., 2017, 10, 387-392.

14. R. Danac; T. Daniloaia; V. Antoci; V. Vasilache; I.I. Mangalagiu; Lett. Drug Des. Discov., 2015, 12, 14-17.

15. A. Rotaru; R. Danac; I. Druta; G. Drochioiu; I. Cretescu; Rev. Chim. (Bucharest, Romania), 2005, 56(2), 179-183.

16. C.M. Al Matarneh, C.I. Ciobanu; I.I. Mangalagiu; R. Danac; J. Serb. Chem. Soc., 2016, 81(2), 133-140.

17. C.M. Al Matarneh; I.I. Mangalagiu; S. Shova; R. Danac; J. Enz. Inhib. Med. Chem., 2016, 31(3), 470-480.

18. A.-M. Olaru; V. Vasilache; R. Danac; I.I. Mangalagiu; J. Enz. Inhib. Med. Chem., 2017, 32(1), 1291-1298.

19. S. Mondal; A. Maity; R. Paira; M. Banerjee; Y.P. Bharitkar; A. Hazra; S. Banerjee; N.B. Mondal; Tet. Lett., 2012, 53, 6288-6291.

20. C.M. Al Matarneh; C.I. Ciobanu; M.O. Apostu; I. I. Mangalagiu; R. Danac; C.R. Chimie, 2018, 21(1), 1-8.

21. C.M. Al Matarneh; M.O. Apostu; I.I. Mangalagiu; R. Danac; Tetrahedron, 2016, 72, 4230-4238.

22. R. Danac; L. Leontie; A. Carlescu; G.I. Rusu; Mat. Chem. Phys., 2012, 134(2-3), 1042-1048.

23. I. Druta; R. Danac; M. Ungureanu; G. Drochioiu; Ann. Pharm. Fr., 2002, 60, 348351.

24. K.M. Khan; Z.S. Saify; Z.A. Khan; M. Ahmed; M. Saeed; M. Schick; H.J. Kohlbau; W. Voelter; Arzneim.-Forsch., 2000, 50(10), 915-924.

25. D.F. Brown; D. Kothari; J. Clin. Pathol., 1975, 28, 779-783.

26. CrysAlis RED, Oxford Diffraction Ltd.,Version 1.171.36.32, 2003.

27. O.V. Dolomanov; L.J. Bourhis; R.J. Gildea; J A.K. Howard; H.J. Puschmann; Appl. Cryst., 2009, 42, 339-341.

28. G.M. Sheldrick; SHELXS, Acta Crystallogr. A, 2008, 64, 112-122. 